$\Rightarrow$

\title{
I spy in my nuclei
}

\section{$c$ \\ i-motifs are \\ present in \\ regulatory \\ regions of \\ DNA}

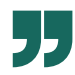

Nature primarily stores genetic information in the right-handed double helices that we know as B-form DNA. However, DNA can also adopt non-helical structures such as the G-quadruplex (G4) motif, wherein four G-rich sequences - present in one or more DNA strands - associate through Hoogsteen-type hydrogen bonding. Another quadruplex structure is the intercalated motif (i-motif) built from a stack of intercalated CC pairs, which, when hemiprotonated, are stabilized by additional $\mathrm{N}^{+}-\mathrm{H} \bullet \bullet \mathrm{N}$ interactions. Although we have learnt much about the biological role of G4s by visualizing them in cells, we have little direct evidence that $\mathrm{i}$-motifs exist in vivo. Writing in Nature Chemistry, a team led by Daniel Christ and Marcel Dinger describe a human antibody fragment that recognizes i-motifs with high selectivity and affinity in the nuclei of human cells.

The team screened the Garvan-2 human single-chain variable fragment library for antibodies that bind the i-motif in human telomeres (hTelo i-motif; telomeres are repetitive nucleotide sequences at the ends of chromosomes), naming

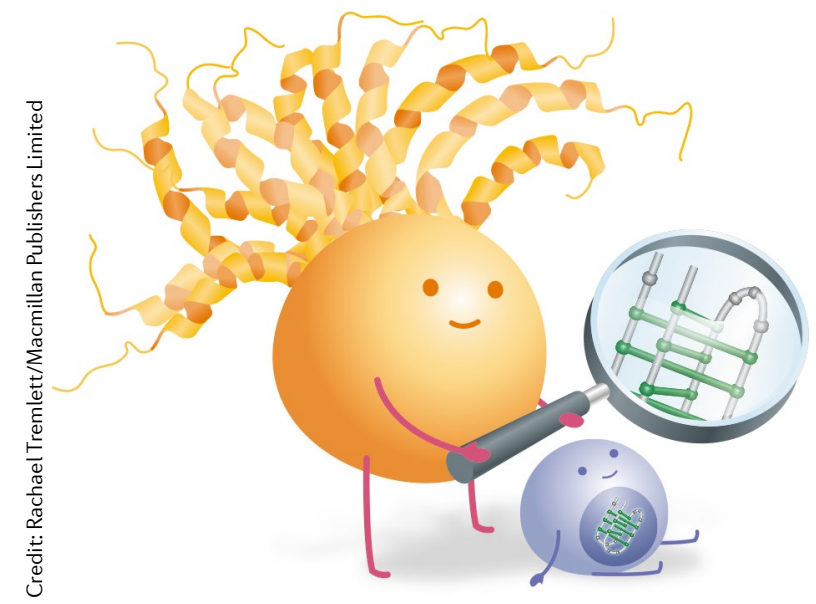

the leading candidate 'iMab. "We used an in vitro technology called phage display to isolate the iMab antibody, allowing for a great deal of control over selection conditions to maximize specificity," explains Christ. "We then used techniques such as biosensor measurements and fluorescence microscopy to further confirm that iMab binds to the i-motif DNA structure."

The interaction between iMab and the hTelo i-motif does not perturb the i-motif, the characteristic circular dichroism spectrum of which is unaffected by kinetically $\left(k_{\mathrm{d}}=1.9 \times 10^{-3} \mathrm{~s}^{-1}\right)$ and thermodynamically $\left(K_{\mathrm{D}}=59 \mathrm{nM}\right)$ favourable binding. The antibody also recognizes i-motifs present in six other genes tested in vitro, including those encoding the proto-oncogenes MYC (a transcription factor) and RET (a growth factor receptor). The strength of these interactions contrasts the lack of affinity iMab has for several proteins and nucleic acids, including duplex DNA, hairpin DNA and microRNA. Importantly, iMab does not bind truncated (non-quadruplex) hTelo genes or G4s, the latter motifs often being found near complementary G-rich sequences. "The challenge in generating the i-motif antibody, which was really the critical step of the study, was not so much about binding but about specificity," explains Christ.

Having demonstrated the utility of iMab in vitro, the real test was to see if the antibody could recognize i-motifs in human cell lines. Indeed, immunofluorescence staining using iMab uncovered i-motifs in the nuclei of three human cell lines, with the emission being strongest in cells expressing folded i-motif oligonucleotides. So, i-motif structures do indeed form in the nuclei of human cells and can be detected by iMab.

With their new i-motif probe in hand, Christ, Dinger and colleagues sought to learn more about i-motifs in vivo. First, using iMab staining and flow cytometry, they showed that levels of $\mathrm{i}$-motifs in dividing cells are highest just before DNA replication. Second, the team lowered the $\mathrm{pH}$ of the cell culture medium and noticed an increased presence of i-motifs, reflecting the importance of hemiprotonation in stabilizing CC pairs. Third, co-staining cells with iMab and a marker of telomeres or a key transcription factor, followed by stimulated emission depletion microscopy, revealed that i-motif structures form in telomeres and promoters. Thus, i-motifs are present in regulatory regions of DNA.

The discovery of this highly specific antibody for i-motifs and the confirmation that these structures exist in vivo will no doubt drive the field forward. The next key task "Is to map precisely where in the genome these i-motifs occur and how and whether they are regulated during development," explains Dinger. Given that i-motifs are present in key regulatory regions of DNA, including those that can be altered in diseases, Dinger notes "As we understand more about the whereabouts of these elements, it will also be interesting to see whether we can identify potentially damaging mutations in them that could explain the basis of some genetic disorders."

Katharine H. Wrighton

ORIGINAL ARTICLE Zeraati, M. et al. I-motif DNA structures are formed in the nuclei of human cells. Nat. Chem. https://doi.org/10.1038/ 441557-018-0046-3 (2018) FURTHER READING Neidle, S. Quadruplex nucleic acids as targets for anticancer therapeutics. Nat. Rev. Chem. 1, 0041 (2017) 